\title{
Aprendizaje servicio en la implementación de política pública para la infancia
}

\author{
Leticia Ivonne López \\ Universidad de Monterrey, México
}

\section{Resumen}

Las Casas Hogar son organizaciones públicas y privadas que tienen como objetivo la atención de niñas y niños que han sido separados de la familia por abuso o violencia. Esta separación se realiza por la autoridad estatal correspondiente o de forma voluntaria por parte de alguno de los miembros de la familia. En 2011 se publicó una Ley para regular las Casas Hogar en Nuevo León, México. Cabe señalar que de las 45 Casas Hogar privadas que existían en ese momento, solamente 3 cumplían con los requisitos para recibir la licencia de operación. A partir de este momento se crearon mecanismos gubernamentales para apoyar su profesionalización, con la finalidad de asegurar la protección de los derechos de la infancia institucionalizada. Fue en este proceso que la Universidad de Monterrey se integra como un actor más para apoyar a cumplir con algunos requisitos para la certificación, y los estudiantes a través de la materia Teorías de Aprendizaje contribuyeron a la consolidación del área de Psicopedagogía de 8 Casas Hogar mediante el desarrollo de Manuales Pedagógicos enfocados a las necesidades educativas de los niños que habitan estas instituciones. Este artículo busca describir y analizar las relaciones entre los actores que contribuyeron a la educación para la ciudadanía de los estudiantes universitarios al implementar su proyecto de aprendizaje servicio como parte de este proceso de políticas públicas.

\section{Palabras clave}

Institucionalización, actores, ciudadanía, proceso.

Fecha de recepción: 22/II/2018

Fecha de aceptación: 22/III/2018 


\title{
Service learning in in the implementation of public policy for children
}

\begin{abstract}
Las Casas Hogar are public and private organizations that aim to care for children who have been separated from the family due to abuse or violence. This separation is carried out by the corresponding state authority or voluntarily by one of the family members. In 2011, a Law was published to regulate Casas Hogar in Nuevo León, Mexico. It should be noted that of the 45 private Casas Hogar that existed at that time, only 3 met the requirements to receive the operating license. Governmental mechanisms were created to support their professionalization, with the aim of ensuring the protection of the rights of institutionalized children. It was in this process that the University of Monterrey is integrated as one more actor to support to meet some requirements for certification, and students through the subject Learning Theories contributed to the consolidation of the area of Psicopedagogy of 8 Casas Hogar through the development of Pedagogical Manuals focused on the educational needs of the children who inhabit these institutions. This article seeks to describe and analyze the relationships between the actors that contributed to citizenship education for university students by implementing their service learning project as part of this public policy process.
\end{abstract}

\section{Keywords}

Institutionalization, actors, citizenship, process. 


\section{Introducción}

Contribuir a la reflexión sobre la participación en la implementación de políticas públicas por parte de proyectos de aprendizaje servicio es el objetivo fundamental de esta investigación. El tema de la regulación y profesionalización de las Casas Hogar ha ocupado un lugar importante en la agenda del Gobierno de Nuevo León, en México. Estas organizaciones atienden a menores que han sido separados de su familia por una autoridad estatal o de forma voluntaria cuando un miembro de la familia los ingresa por situaciones de pobreza, abuso o violencia. En Diciembre del 2008 se presentó un caso de abuso, secuestro y trata de menores por parte de una Casa Hogar, denominada Centro de Adaptación y Reintegración Familiar A.C. Con esta evidencia se movilizaron actores privados y públicos, así como los medios masivos de comunicación para darle cobertura y solución al caso. A partir del recuento de los hechos, a posteriori, la protección de la infancia institucionalizada se convirtió en uno de los objetivos principales del organismo gubernamental que tenía como función la protección de la familia en el Estado. Estos antecedentes llevaron a publicar la primera Ley en México para regular a las Casas Hogar en el 2011 y en el 2012 se les entregaron las primeras licencias de operación por parte del Gobierno Estatal, para posteriormente continuar con la certificación de estas instituciones.

Las Casas Hogar tenían que cumplir con una serie de requisitos, entre ellas contar con un área de psicopedagogía para apoyar el proceso educativo y formativo de los niños y adolescentes que habitaban este tipo de organizaciones. Es por lo anterior que se le propuso a la profesora Bertha Adriana Díaz Delgado, que impartía la materia Teorías del Aprendizaje, desarrollar Manuales para el acompañamiento pedagógico para algunas de las Casas Hogar que estaban en proceso de certificación. Por lo que este proyecto de aprendizaje servicio es un ejemplo que se puede vincular a la academia a resolver problemas sociales mediante la participación en políticas públicas del sector público. Como mencionan Giorgetti et al (2007), podríamos considerar que a partir de los proyectos de aprendizaje servicio se puede conectar a los ciudadanos entre sí y con instituciones estatales, modificando la división público-privado de una forma novedosa y generando una articulación más compleja que permite construir nuevas normas y lealtades que promueve el desarrollo del capital social.

\section{Marco Teórico}

Se considera el estudio de las instituciones para explicar el concepto de institucionalización de la infancia, que en este caso es la población que habita en Casas Hogar y como éstas junto con otros actores colectivos e individuales contribuyen a la protección de sus derechos. Las instituciones han sido estudiadas desde diferentes enfoques. Autores como Peters (2003) mencionan hasta siete institucionalismos: sociológico, de regímenes internacionales, grupos de interés como instituciones, normativo, 
de elección racional, historico y empírico.

El enfoque propuesto para este trabajo de investigación es el de Giddens (1998), que indica que se reproducen las formas de conducta social a través del tiempo y espacio, enfatizando el poder de las normas y su influencia en el comportamiento de los individuos como parte de su concepto de estructura. En esta investigación se aborda parte del análisis desde el institucionalismo sociológico, porque explica la relación entre los actores sociales y su contexto, en el que se espera que una Casa Hogar adopte la cultura de su entorno determinada por una Ley Estatal como base de su legitimidad.

Giddens (1998) otorga al Estado un papel importante como regulador de las relaciones sociales y los derechos de propiedad. Considera que la estructura solo existe como hábitos que se actualizan en las acciones. Desde antes del 2006, las prácticas sociales realizadas al interior de las Casas Hogar para el cuidado y protección de los menores que habitaban en ellas no habían sufrido cambios radicales y la intervención de los organismos gubernamentales era casi nula.

\section{La estructura definida por Giddens} (1998) como el conjunto de reglas y recursos que se organizan como propiedades que se manifiestan en prácticas sociales, brinda fuerza a este proceso experimentando por las Casas Hogar a partir del 2011 con la publicación de la nueva normativa. El análisis se llevará a cabo a partir de los individuos, a los que llamaremos agentes, quienes son los que cambian las instituciones. Los límites impuestos por éstas en las decisiones individuales son determinantes.

Fue un grupo de personas pertenecientes al gobierno estatal que por el contexto que se estaba viviendo en el 2008 con el caso de desaparición de unos niños del Centro de Adaptación y de Integración Familiar A.C.

empezaron a gestionar acciones para crear una ley que regulara a las Casas Hogar en Nuevo León; con la intención de prevenir un evento similar al ocurrido en Sonora (la explosión en la Guardería $A B C$, en donde fallecen bebés y niños al cuidado de ésta), además de proteger los derechos humanos de los menores que habitan dentro de las Casas Hogar de esta región.

Por otro lado, North (1990) menciona tres estructuras que componen el marco institucional: la política, que especifica la manera en la que nos desarrollamos y conseguimos nuestra función dentro de la sociedad; la de derechos de propiedad, que establece los incentivos económicos formales y la social, es decir, las normas y convenciones, que definen cómo nos relacionamos. Este enfoque se reafirma con el concepto de sistema de Giddens (1998), que comprende relaciones reproducidas entre actores 0 colectividades, organizadas como prácticas sociales regulares. En este caso, el tipo de estructura a considerar en la institucionalización de la infancia en Nuevo León es la social, porque si bien las reglas formales (leyes) que una sociedad establece reflejan su herencia, son las restricciones 
informales, o normas de comportamiento, convenciones y códigos autoimpuestos los que fijan principalmente la estructura en la que vivimos. Y éstas empezaron a ser evaluadas y revisadas por el gobierno estatal a través del Consejo Estatal de Adopciones en el 2006, cuando sus miembros empezaron a visitar las Casas Hogar y contrataron a una persona para este fin, para después en el 2007 solicitar un estudio a la Universidad Autónoma de Nuevo León para evaluar la salud de la infancia que habitaba en ese momento las Casas Hogar con la finalidad de empezar a observar si las prácticas sociales realizadas al interior de estas instituciones estaban protegiendo los derechos de los menores que se encontraban viviendo en esos lugares.

Es importante considerar que en las instituciones gubernamentales como el DIF Nuevo León, el comportamiento de los individuos se restringe por la función de cada actor dentro de esa estructura. Es decir, el mismo actor en situaciones diferentes tendrá comportamientos diferentes. Un actor político o normativo toma una determinada función o identidad y se pregunta qué debe hacer de acuerdo a ésta.

En sociología, las instituciones son prácticas reconocidas que consisten en funciones fáciles de identificar, asociadas con reglas o convenciones que gobiernan las relaciones entre quienes tienen estas funciones. Ciertas acciones sociales se dan por el contexto en el que se encuentran las personas que se están relacionando y las cogniciones compartidas definen lo que tiene determinado significado y qué acciones son posibles (Powell y DiMaggio, 1999).

La teoría de la estructuración de Giddens (1998) hace énfasis en la acción humana a través de un agente que tiene la capacidad de incidir en su grupo social, por lo que la Dirección de Protección de la Infancia y la Familia del DIF Nuevo León empezó a cuestionar su responsabilidad de hacer algo ante los sucesos del 2008 y 2009; en donde se afectó la vida e integridad de menores. Sin embargo, la única entidad que estaba ligada al DIF Nuevo León en ese momento para realizar acciones de protección a la infancia institucionalizada era el Consejo Estatal de Adopciones, organismo que había empezado a generar proyectos a favor de esta población en el 2006. Es por esta razón, que fue esta instancia la que inició el proceso de profesionalización y regulación de las Casas Hogar en Nuevo León y en donde la Universidad de Monterrey fue un actor más de este proceso.

\section{Metodología}

Esta es una investigación analítica con una perspectiva sociológica del proceso de institucionalización de las Casas Hogar en Nuevo León. Este enfoque sociológico desde una perspectiva accionista tiene dos principios y se relaciona con la integración social de la teoría de la estructuración, que hace referencia a la reciprocidad de prácticas sociales de carácter autónomo y comunicativo entre los individuos en un tiempo y espacio determinados. 
En primer lugar, por medio de las relaciones sociales, los actores establecen patrones a través de los cuales se vinculan entre sí. Así el estudio de las relaciones sociales remplaza al estudio de la sociedad como objeto central de la sociología (Zapata, 2005). Es por medio de las relaciones sociales que se pueden comprender prácticas sociales que se presentan en un determinado espacio y tiempo, para comprender su conformación.

En segundo lugar, a través de los significados que los actores dan a su práctica social, definiendo la realidad en la que se encuentran no en términos de lo que los actores dicen sino en términos de su reacción ante la hipótesis de investigación. Cuando los grupos difieren con respecto a la hipótesis, el investigador debe redefinirla o concluir que el proceso analizado posee otros significados que tienen que ser articulados en nuevas hipótesis. Intenta responder cómo la sociedad se construye a partir de la acción de sus actores que se encuentran participando en algún proceso social. Esto se relaciona con el concepto de consciencia del agente de Giddens (1998), que se refiere al registro reflexivo de la acción y la conciencia práctica; así como a su capacidad de registrar su entorno físico y social de su vida social y utilizarla como soporte de su seguridad ontológica.

Se privilegiaron los métodos cualitativos, debido a que éstos enfatizan el proceso.
En el 2001 inicié por iniciativa personal una fundación llamada Pequeños

Gigantes Mexicanos A.C., que brinda servicios de apoyo a las Casas Hogar en Nuevo León, y a través de la cual he sido parte del proceso de institucionalización de estas organizaciones desde entonces. Por otro lado, provengo de una familia en la que mis abuelos, después de una situación de abandono por parte de sus padres, fueron otorgados en adopción con personas que no eran parte de su familia nuclear ni extensa.

Esto ha provocado que la visión con la que he observado durante el proceso de investigación se encuentre cargada de pre-nociones obtenidas por la experiencia personal. Por ello, ontológicamente he identificado que tengo una visión un tanto socialconstructivista, ya que procuré considerar las diferentes perspectivas de los actores participantes en el proceso observado.

Esta visión propone que los individuos y la sociedad perciben como realidad lo que no es más que una construcción, una creación de la interacción social entre esos individuos y los grupos. $Y$ desde esta perspectiva, se le da mayor importancia a los significados, reflexiones, discursos y comprensión de los actores en relación con el proceso social en la que se encuentran.

Es por lo anterior que este método fue el más adecuado para esta investigación, porque la información recolectada se obtuvo de la voz de los actores que participaron en el proceso de institucionalización y desde ahí se 
inició la construcción del modelo de protección de la infancia institucionalizada en Nuevo León

En este caso se tenía como objetivo de investigación analizar las interacciones entre la profesora y sus alumnos con el proceso de regulación y profesionalización de las Casas Hogar en Nuevo León con el DIF Nuevo León como parte de un proceso de educación para la ciudadanía. María Luisa Tárres (2001) menciona que los métodos cualitativos ponen énfasis en la visión de los actores y el análisis contextual en el que éstos se desarrollan, centrándose en el significado de las relaciones. Por lo que fueron los métodos cualitativos los más adecuados para capturar esas relaciones y la subjetividad de los actores. Los métodos cuantitativos no consideran los detalles, los cuales para este análisis son relevantes porque permiten conocer las funciones de los actores mencionados en el problema de investigación. Son los métodos cualitativos los que conceptualizan la realidad, que en este caso se requiere para que la información se convierta en un análisis del proceso de institucionalización de las Casas Hogar de Nuevo León.

Se realizaron entrevistas a las Casas Hogar beneficiarias del proceso y a 10 estudiantes que estuvieron participando en la evaluación de los Manuales de Pedagogía para identificar el tipo de actor que este grupo tuvo en el proceso de institucionalización que se tenía en Nuevo León durante el 2016 en que fue desarrollado este proyecto. Ahora bien, desde el paradigma socioconstructivista es la comunidad receptora la que valida los resultados de investigación (Easterby-Smith et. al, 2012) lo cual creo se logró plenamente al entregarle a la comunidad un Manual Normativo para Casas Hogar (López, 2015) y los 8 Manuales de Pedagogía.

\section{Resultados y discusión}

Las prácticas institucionalizadas en las Casas Hogar comenzaron a cambiar paulatinamente a partir del 2006 y la transformación mayor se inició a partir del 2011, cuando el poder de los agentes públicos comenzó a generar un sistema de dominación para la regulación y profesionalización de las Casas Hogar con la finalidad de cumplir con la Convención de los Derechos del Niño ratificada por México en 1989. Así empezaron a integrarse por parte de los agentes rasgos temporales y espaciales de encuentros en procesos de constitución de sentido, dando origen a los siguientes tipos de agentes:

- Agentes protectores, para garantizar que se realicen las acciones necesarias para que sus derechos no sean violados y puedan ejercerlos libremente. Es de donde surgen las reformas y publicaciones de nuevos marcos jurídicos que contribuyan a la protección de los derechos de la infancia en las Casas Hogar y nuevos agentes. Un agente deja de ser tal si pierde la aptitud de producir una diferencia, o sea, de ejercer alguna clase de poder (Giddens, 1998).

- Agentes reguladores. Su responsabilidad es crear los 
mecanismos necesarios para asesorar a estas instituciones, para que puedan realizar los cambios necesarios dentro de su operación para cumplir con la normativa (Cardoza, 2011).

- Agentes cuidadores. Sus acciones están enfocadas a brindar apoyo a las Casas Hogar con la finalidad de mejorar su operación y colaborar para que puedan cumplir con los lineamientos establecidos en el marco jurídico vigente como donativos, crear proyectos, etc.

Posteriormente comenzaron a surgir nuevos agentes públicos y privados, con nuevas prácticas sociales, como comités, iniciativas y formalización de redes de apoyo que nacen de este proceso relacional. Y la Universidad de Monterrey a través de la materia de Teorías del Aprendizaje fue un actor más en este proceso que surge y se suma al mismo. En este caso el aprendizaje servicio realiza un aporte tanto para la generación de las capacidades para participar como en la promoción de experiencias que le brinden a los estudiantes experiencias reales de oportunidades de participar.

Con este proceso de institucionalización se iniciaron nuevas formas de socialización durante las interacciones entre los agentes que se categorizaron de la siguiente forma:

- Socialización colaborativa. Ambos van modificando sus acciones por el intercambio de estímulos que se presentan en la relación. En este tipo de socialización es donde se generan los cambios en las prácticas sociales que se habían estado realizando a través del tiempo.

- Socialización legalista. Se expresa entre dos agentes, en donde uno de ellos tiene la autoridad legal de regular y supervisar al otro durante la interacción.

- Socialización soporte. Se manifiesta en la interacción de dos agentes, cuando uno de ellos apoya mediante conocimiento, recurso humano, donativos en efectivo o especie, acompañamiento, asesoría, programas y proyectos de apoyo al otro. Estas nuevas formas de socialización dieron origen a nuevos agentes privados como fueron las redes de apoyo a Casas Hogar, conformadas por instituciones $\mathrm{y} / \mathrm{o}$ individuos interesados en este tipo de población.

Al interior de las Casas Hogar también existen relaciones entre otros actores colectivos e individuales, pero los recursos de autoridad se distribuyen de forma diferente en cada Casa Hogar, dependiendo de su estructura organizacional y la forma de operación. Un agente es capaz de desplegar un espectro de poderes causales, porque una acción nace de la aptitud del individuo para producir una diferencia en un estado de cosas o curso de sucesos preexistentes.

La movilización de influencia que empezaron a generar los agentes colectivos e individuales públicos sobre las Casas Hogar en Nuevo León dieron origen a una nueva distribución de los recursos de significación y legitimación que se empezaron a intercambiar 
durante las interacciones obligadas por el cumplimiento de la nueva normativa. Estos recursos son propiedades estructurales de los sistemas sociales, que los agentes colectivos e individuales utilizan y reproducen en cada interacción. Recordando que el poder mismo no es un recurso, los recursos son medios a través de los cuales se ejerce poder, como un elemento de rutina de la actualización de una conducta en una reproducción social. El poder en sistemas sociales que disfrutan de cierta continuidad en tiempo y espacio presupone relaciones regularizadas de autonomía y dependencia entre actores 0 colectividades en contextos de interacción social, pero todas las formas de dependencia ofrecen ciertos recursos de los cuales, los subordinados pueden influir sobre las actividades de sus superiores.

Y es aquí donde la participación del equipo de Teorías de Aprendizaje de la Universidad de Monterrey se puede pensar como una instancia de poder que considera modos alternativos al control social dentro de una de las áreas de la Casa Hogar, presentando una socialización de soporte porque brindaron material que era parte de los requisitos a cumplir para la certificación que el Gobierno Estatal estaba solicitando y como agentes cuidadores porque como se menciona anteriormente, a diferencia de los agentes protectores y legalistas, los cuidadores contribuyen a apoyar el cumplimiento de la normativa vigente.

\section{Conclusiones}

Surge un nuevo modelo de protección a la infancia institucionalizada en Nuevo León, con prácticas sociales que empiezan a reproducirse entre los agentes involucrados en el proceso de regulación y profesionalización de las Casas Hogar. Además, emerge un nuevo sistema de relaciones con acciones que comienzan a regularse por la normativa, es decir, inician un proceso de institucionalización como resultado de las practicas que las Casas Hogar organizan de forma recursiva en su actuar cotidiano.

Es en este proceso donde se integra la Universidad de Monterrey con posibilidad en participar en espacios micro, para potenciar la articulación con nuevas organizaciones que actúa en la arena política permitiendo realizar acciones conjuntas a favor de problemáticas a favor del bien común como parte de una nueva normativa.

La integración de las nuevas prácticas sociales en este sistema de protección a la infancia institucionalizada sucedió en dos niveles de interacción, de acuerdo a la teoría de la estructuración que Giddens (1998) nombra como integración social (micro) e integración sistémica (macro) que también se analizaron en los apartados anteriores, teniendo como resultado del análisis un sistema de protección a la infancia institucionalizada que empezó a constituirse como tal mediante el aumento de sus interacciones y transformación de sus acciones cotidianas. El autor incluye ambos niveles (micro y macro) en donde las 
relaciones son reproducidas tanto por individuos y colectividades que interactúan entre sí. Así que mientras los colaboradores de una Casa Hogar interactúan para realizar la operación cotidiana de la institución y modifican algunas de sus acciones para cumplir con la normativa.

La participación concreta fue la entrega de ocho Manuales de Pedagogía para las Casas Hogar en Nuevo León (Asociación de Enfermeras y Trabajadores Sociales AC; Retiro Juvenil AC; Manantial de Amor AC; Casa Hogar Douglas; Bethany AC; EI Refugio de Monterrey AC; El Oasis de los Niños AC y Ministerios de Amor AC) mediante la participación de 60 estudiantes y guiados por su profesora de Teorías del Aprendizaje. Por lo que en el marco de la rearticulación de las relaciones estado/sociedad civil, la relación institución educativa/comunidad plantea posibles relaciones entre actores involucrados no solo enfocados a la responsabilidad educativa sino desarrollando capacidades para la participación ciudadana. Los miembros de la materia de Teorías del Aprendizaje fueron agentes cuidadores con una socialización colaborativa que durante el proceso construyeron ciudadanía. Porque para realizar este proyecto, tuvieron que recopilar, exigir y distribuir información, permitiéndoles acercarse a la situación real de una política pública y su implementación.

\section{Referencias bibliográficas}

Cardoza, R. (2011). Articulación del trabajo en redes de política pública a través de la solución efectiva de conflictos. Caso: instituciones asistenciales de niñas, niños y adolescentes en Nuevo León. México: Tecnológico de Monterrey.

Easterby-Smith, M., Thorpe, R. and Lowe, A., (2002), Management Research: An Introduction. London: Sage Publications

Giddens, A. (1998). La Constitución de la sociedad. Bases para la Teoría de la Estructuración. Buenos Aires:

Amorrotu.

Giorgetti, D.A.; Baioni, P.; Gimelli, A. ; Malacrida, M.G.; Penjerek, M.M. (2007). Educar en la Ciudadanía. El aporte del aprendizaje-servicio. Buenos Aires: CLAYSS.

López, L. (2015). Manual Normativo para Casas Hogar. Guía de Operación basado en el marco jurídico para la protección de los Derechos de las Niñas, Niños y Adolescentes. México: DIF.

North, D. (1990) Institutions, Institutional Change and Economic performance. Cambridge: Cambridge University Press.

Peters, B. G. (2003). El nuevo institucionalismo: Teoría institucional en ciencia política. Barcelona: Gedisa.

Powell, W.; DiMaggio, P. (1999). El nuevo institucionalismo en el análisis organizacional. México: Fondo de Cultura Económica.

Tárres, M.L. (2001). Observar, escuchar y comprender. Sobre la 
tradición cualitativa en la investigación social. México: Colegio de México.

Zapata, Francisco (2005). Cuestiones

de Teoría sociológica. México: Colegio

de México. 